\title{
Corpus
}

4 | 2005

Les corpus politiques : objet, méthode et contenu

\section{Damon Mayaffre - Paroles de président. Jacques Chirac (1995-2003) et le discours présidentiel sous la Vème République. Paris : Champion, 2004, 292 pages $(50 €)$.} Jacques Guilhaumou

\section{(2) OpenEdition}

\section{Journals}

Édition électronique

URL : http://journals.openedition.org/corpus/322

DOI : $10.4000 /$ corpus.322

ISSN : $1765-3126$

Éditeur

Bases; corpus et langage - UMR 6039

Édition imprimée

Date de publication : 1 décembre 2005

Pagination : 218-221

ISSN : 1638-9808

Référence électronique

Jacques Guilhaumou, «Damon Mayaffre - Paroles de président. Jacques Chirac (1995-2003) et le discours présidentiel sous la Vème République. Paris : Champion, 2004, 292 pages (50€). », Corpus [En ligne], 4 | 2005, mis en ligne le 05 septembre 2006, consulté le 23 septembre 2020. URL : http:// journals.openedition.org/corpus/322 ; DOI : https://doi.org/10.4000/corpus.322

Ce document a été généré automatiquement le 23 septembre 2020.

(c) Tous droits réservés 


\title{
Damon Mayaffre - Paroles de président. Jacques Chirac (1995-2003) et le discours présidentiel sous la Vème République. Paris : Champion, 2004, 292 pages $(50 €)$.
}

\author{
Jacques Guilhaumou
}

1 Après son étude exemplaire sur le discours de gauche et de droite pendant l'entredeux-guerres dans le champ de la lexicométrie appliquée à un très grand corpus, Damon Mayaffre élargit notre horizon méthodologique, en matière de lexicologie quantitative, par une approche logométrique du discours d'un homme politique, Jacques Chirac, qu'il met en perspective au sein du discours présidentiel entre 1958 et 2003. La logométrie s'entend ici comme un ensemble de traitements documentaires et statistiques du texte qui prend en compte aussi bien les lemmes, les structures grammaticales que les formes graphiques. Elle mobilise à la fois des outils de synthèse (analyse factorielle des correspondances, analyse arborée) et des instruments de description (lexique commun, spécificités lexicales et grammaticales).

Il s'agit alors de décrire la surface discursive d'un très grand corpus textuel de 1800 discours et 4,4 millions d'occurrences dans toutes ses dimensions - graphiques, lemmatisées et grammaticalisées -, sans pour autant réduire le texte à du discours. En effet, l'interprétation des textes, sous l'angle de la quantification, ne peut se réduire à la seule mise à l'épreuve d'hypothèses sur la base des conditions de production du discours, mais relève avant tout de pratiques herméneutiques et heuristiques qui permettent d'élargir l'apport documentaire des résultats quantifiés à la reconnaissance de phénomènes inédits tant sur le plan historique que linguistique. 
3 La première partie de l'ouvrage dresse un bilan du discours présidentiel sous la Vème république. Le lexique commun à de Gaulle, Pompidou, Giscard, Mitterrand et Chirac, hiérarchisé par les sémantismes de France, Europe et Monde au profit d'une phraséologie patriotique, montre un nombre limité de termes politiques sémantiquement forts, certes avec un moindre degré chez de Gaulle. Ainsi le trait linguistique le plus saillant renvoie plutôt à des considérations grammaticales : la transition, au-delà des variations individuelles, d'un discours nominal (récurrence des déterminants, noms et adjectifs) à un discours verbal (récurrence des pronoms, des verbes et des adverbes), au tournant des années 1980. A une relative homogénéité de ce qui se dit s'ajoute donc, dans une sorte d'homogénéité montrée, un dire ( "Je vous dis que ») à forte valeur performative jusqu'à l'exhibition d'un véritable «narcissisme linguistique». Le discours de la lutte, du combat sombre dans le verbalisme, non sans une certaine efficacité réceptive. La présidence de Mitterrand contribue de façon décisive à une telle mise en scène du dire et de son énonciateur. (Pourquoi? Au-delà du triomphe d'une pensée unique qui n'a plus besoin de s'expliciter mais a besoin d'être incarnée, deux hypothèses sont avancées pour expliquer cette évolution: l'évolution institutionnelle de la Vème République avec l'invention de la cohabitation qui dissipe l'affrontement politique au profit de l'affrontement de personnes; l'influence de la télévision qui médiatise désormais, seule, le discours présidentiel et favorise la personnification du débat).

La seconde partie de l'ouvrage aborde alors le discours de Chirac proprement dit sur la base d'une telle absence de particularités saillantes dans le dit présidentiel lui-même et au plus grand profit des artifices performatifs. Il est vrai que Chirac excelle dans le neutre d'un dire illocutoire. Usant d'une identité lexicale plutôt évanescente par le fait d'associer les thématiques au factuel, donc à la variation conjoncturelle, Chirac renforce le discours verbal de mise à distance de tout contenu nominal par la sur utilisation des adverbes, qui plus est sur le mode de l'évidence présupposée, à l'exemple des usages répétés de "naturellement ». D'autres processus de même type sont tout aussi visibles à la lecture des résultats quantifiés. Ainsi Damon Mayaffre peut affirmer que «Comme l'usage des adverbes, l'usage exclusif du présent de l'indicatif prouve l'obsession du président de marquer par sa présence le discours, et sa volonté de donner à son énoncé un potentiel réflexif grâce auquel non seulement le texte renvoie à son émetteur, mais lie le destinataire à l'énonciateur "(p.117). Peu enclin aux références idéologiques, Chirac adopte une posture rhétorique sur la base des effets illocutoires de sa parole prononcée.

5 Au fil du temps, le discours de Chirac montre certes une variation thématique, par une oscillation entre des considérations dominantes en politique intérieure ou en politique étrangère, et quelques événements lexicaux à l'exemple de la question du chômage en 1995 (et de l'insécurité en 2002). Mais l'intérêt de la démarche logométrique renvoie surtout au constat de temps discordants dans une évolution, à l'exemple de l'année 2003 qui renoue avec le repli sur la politique étrangère de 1998.

Bien sûr le lecteur attendait avec une certaine impatience de voir comment Damon Mayaffre allait rendre compte du lien entre Chirac et Jospin, au regard de l'échec de ce dernier en 2002. Tout d'abord Damon Mayaffre nous propose une démarche comparative qui tend à accentuer les traits spécifiques du discours chiraquien. Ainsi le discours de Chirac «se caractérise par son indigence de noms (écart réduit de -38 par rapport à Jospin) comme si le président, dans le débat, avait peur des mots-pleins porteurs de sens. En revanche le parler de Chirac se fait remarquer par la sur- 
utilisation d'adverbes $(+37)$ et de pronoms personnels $(+32) »($ p. 226). Ainsi la vacuité idéologique du discours chiraquien s'en trouve renforcée tant le contenu sémantique des paroles prononcées lui importe peu au regard de leur énonciation et donc du dire ainsi mis en scène. Ainsi s'agit il $d^{\prime}$ '« un discours moins remarquable par l'inanité fluctuante de son dit que par la force permanente de ce qu'il représente ».

Reste à comprendre l'échec de Jospin, qui ne peut se réduire au fait du constat du rapprochement du vocabulaire des deux hommes politiques, et donc d'un certain affadissement du clivage droite-gauche. A ce propos, l'approche logométrique montre une position extraordinaire de Jospin2002 dans le graphe arborée, présenté page 231, par le fait d'une rupture linguistique : il apparaît un discours mutant avec des mots de Chirac, à distance du discours usuel de gauche, donc inaudible pour une partie de l'électorat. S'il est certain que Jospin devait se soumettre à la loi du genre discursif électoral, il n'en reste pas moins que sa renonciation massive aux noms politiques, et au mot « politique » lui-même, au profit d'un discours verbal l'a entraîné sur un terrain qui ne convenait guère à lui-même et aux élécteurs de gauche.

8 Damon Mayaffre, d'une étude à l'autre, nous interpelle sur la capacité d'innovation d'une démarche lexicale d'ordre quantitatif qui permet d'appréhender le texte sans parti pris, donc dans le fait même de sa description. Avec son premier travail, il montre que l'abord lexicométrique des très grands corpus permet de circonscrire le contexte dans le texte lui-même, à l'encontre d'une appréhension limitée d'une part discursive d'un corpus textuel sur la base d'un référent préalablement défini, sous le label de conditions de production. Cette nouvelle étude interroge plus avant le lien consubstantiel entre texte et discours, au regard d'une herméneutique matérielle. Elle s'ouvre sur une investigation de nature essentiellement philologique, qui conteste donc qu' " un texte pourrait préexister à l'énonciation qui va le transformer en discours, alors même qu'il se trouve défini comme expression et produit » (François Rastier). 